\title{
Different Results of IgE Binding- and Crosslinking-Based Allergy Tests Caused by Allergen Immobilization
}

\author{
Yoshimi Okamoto-Uchida, ${ }^{a}$ Ryosuke Nakamura, ${ }^{*, a}$ Yumiko Matsuzawa, ${ }^{a}$ Megumi Soma, ${ }^{b, c}$ \\ Hiroshi Kawakami, ${ }^{c}$ Akiko Ishii-Watabe, ${ }^{d}$ Tomoko Nishimaki-Mogami,${ }^{b}$ Reiko Teshima, ${ }^{b}$ and \\ Yoshiro Saito $^{a}$ \\ ${ }^{a}$ Division of Medicinal Safety Science, National Institute of Health Sciences; 1-18-1 Kamiyoga, Setagaya-ku, Tokyo \\ 158-8501, Japan: ${ }^{b}$ Division of Novel Foods and Immunochemistry, National Institute of Health Sciences; 1-18-1 \\ Kamiyoga, Setagaya-ku, Tokyo 158-8501, Japan: ${ }^{c}$ Department of Food Science and Nutrition, Kyoritsu Women's \\ University; 2-2-1 Hitotsubashi, Chiyoda-ku, Tokyo 101-8437, Japan: and division of Biological Chemistry and \\ Biologicals, National Institute of Health Sciences; 1-18-1 Kamiyoga, Setagaya-ku, Tokyo 158-8501, Japan. \\ Received May 11, 2016; accepted July 6, 2016
}

The physicochemical nature of allergen molecules differ from the liquid phase to the solid phase. However, conventional allergy tests are based on the detection of immunoglobulin (Ig)E binding to immobilized allergens. We recently developed an in vitro allergy testing method using a luciferase-reporting humanized rat mast cell line to detect IgE crosslinking-induced luciferase expression (EXiLE test). The aim of the present study was to evaluate the effects of antigen immobilization on the results of different in vitro allergy tests using two anti-ovalbumin (OVA) antibodies (Abs), E-C1 and E-G5, with different properties in the OVAinduced allergic reaction. Both Abs showed clear binding to OVA with an enzyme-linked immunosorbent assay and by BIAcore analysis. However, only E-C1 potentiated EXiLE response for the liquid-phase OVA. On the other hand, OVA immobilized on solid-phase induced EXiLE responses in both E-C1 Ab- and E-G5 Ab-sensitized mast cells. Western blotting of OVA indicated that E-C1 Ab binds both to OVA monomers and dimers, unlike E-G5 Ab, which probably binds only to the OVA dimer. These results suggest that antigen immobilization enhanced IgE crosslinking ability through multimerization of allergen molecules in the solid phase, resulting in an increase in false positives in IgE binding-based conventional in vitro allergy tests. These findings shed light on the physicochemical nature of antigens as an important factor for the development and evaluation of in vitro allergy tests and suggest that mast cell activation-based allergy testing with liquid-phase allergens is a promising strategy to evaluate the physiological interactions of $\operatorname{IgE}$ and allergens.

Key words immunoglobulin E (IgE); allergen; immobilization; in vitro allergy test; luciferase assay; $\operatorname{IgE}$ crosslinking-induced luciferase expression (EXiLE) test

Immunoglobulin ( $\mathrm{Ig}) \mathrm{E}$ is the primary factor for mast cell activation and type I allergic reactions, such as histamine release and production of inflammatory factors. Although immunochemical allergen-specific $\operatorname{IgE}$ tests based on the binding of serum $\mathrm{IgE}$ to an allergen are used worldwide, the results cannot always be translated into a clear diagnosis, especially in the case of food allergies. ${ }^{1,2)}$ The existence of allergen-specific $\operatorname{IgE}$ indicates that the individual has been previously sensitized to the allergen. However, $\operatorname{IgE}$ is not always capable of crosslinking high-affinity $\operatorname{IgE}$ receptors (FceRI) with the soluble allergen because multiple epitopes of an allergen molecule are required. ${ }^{3)}$ Additionally, there is a greater abundance of antigen-specific IgGs in sera than IgE, which may interfere with the binding of $\operatorname{IgE}$ to the antigen. To overcome this blocking effect, excessive amounts of antigens are immobilized in the solid phase of immunochemical allergy tests, such as the radioallergosorbent test and fluoroenzyme immunoassay ImmunoCAP ${ }^{\circledR}$ (CAP test). ${ }^{4,5)}$ However, immobilization may affect the physicochemical nature of the antigens, resulting in discrepancies of the allergenicity of the molecule between in vivo responses and in vitro tests.

We developed a new allergy test, called the IgE crosslinking-induced luciferase expression (EXiLE) test, ${ }^{6-10)}$ which is dependent on the activation of the humanized rat mast cell line RS-ATL8 sensitized with serum IgE of allergic patients. The EXiLE test detects the ability of IgE to crosslink multi- valent antigens in the liquid phase, resulting in a more reliable diagnosis of egg white food allergy than the conventional CAP test. ${ }^{7}$ )

The EXiLE test can be used to determine the effects of the physicochemical nature of allergens on allergy test results. Here, we used two ovalbumin (OVA)-specific IgE monoclonal antibodies (Abs), E-C1 and E-G5, which can and cannot induce mast cell degranulation, respectively. ${ }^{11,12)}$ Using these two antibodies, we aimed to evaluate the effects of antigen immobilization on the results of different in vitro allergy tests and to further elucidate the characteristics of the cell-based allergy test.

\section{MATERIALS AND METHODS}

Reagents The mouse anti-OVA monoclonal IgE Abs E-C1 and E-G5 were purchased from Chondrex, Inc. (Redmond, WA, U.S.A.). Horseradish peroxidase (HRP)-conjugated anti-mouse IgE was purchased from Bethyl Laboratories (Montgomery, TX, U.S.A.). OVA was purchased from SigmaAldrich (St. Louis, MO, U.S.A.). Anti-mouse IgE for EXiLE experiments and for surface plasmon resonance (SPR) experiments were purchased from Bethyl Laboratories and Yamasa Corporation (Tokyo, Japan), respectively.

Cell Culture The rat humanized mast cell line RS-ATL8 was cultured as previously described..$^{7-10}$ Briefly, the cells 
were maintained in minimal essential medium (MEM; Invitrogen, Rockville, MD, U.S.A.) supplemented with $10 \%$ heatinactivated fetal calf serum (FCS; Biowest, Nuaillé, France), penicillin-streptomycin (Invitrogen), $500 \mu \mathrm{g} / \mathrm{mL}$ of geneticin (Invitrogen), and $200 \mu \mathrm{g} / \mathrm{mL}$ of hygromycin B (Invitrogen).

Enzyme-Linked Immunosorbent Assay (ELISA) OVA was diluted to $0.1-10000 \mathrm{ng} / \mathrm{mL}$ with $50 \mathrm{~mm}$ carbonate buffer ( $\mathrm{pH}$ 9.6) and plated into the wells of a 96-well plate (Corning Incorporated, Corning, NY, U.S.A.). After overnight incubation at $4^{\circ} \mathrm{C}$, the plate was washed three times with $0.05 \%$ Tween-20 in phosphate-buffered saline (PBS). For blocking, the plate was incubated with $0.1 \%$ casein/PBS for $1 \mathrm{~h}$ at room temperature and then washed three times with $0.05 \%$ Tween-20/PBS. Anti-OVA Abs were diluted $(1 \mu \mathrm{g} /$ $\mathrm{mL}$ ) with $0.1 \%$ casein/PBS and added to the plate, which was then incubated at room temperature for $2 \mathrm{~h}$. HRP-conjugated anti-mouse IgE diluted with $0.1 \%$ casein/PBS was added and detected using the BD Opt EIA kit (Becton, Dickinson and Company, Franklin Lakes, NJ, U.S.A.) and an ARVOsx 1420 multilabel counter (PerkinElmer, Inc., Waltham, MA, U.S.A.).

SPR Analysis Anti-mouse IgE was diluted to a concentration of $10 \mu \mathrm{g} / \mathrm{mL}$ with $10 \mathrm{~mm}$ sodium acetate $(\mathrm{pH} 5.5)$ and immobilized on a CM5 biosensor chip (GE Healthcare, Buckinghamshire, U.K.) using an amine coupling kit (GE Healthcare) at a density of $c a .15000$ resonance units. E-C1 and E-G5 anti-OVA Abs were diluted with $\mathrm{HBS}^{-\mathrm{EP}^{+}}$running buffer (GE Healthcare) and captured, whereas nothing was captured on the reference flow cells. Diluted OVA $(0,8,24$, $72,216,648 \mathrm{~nm})$ was injected for $180 \mathrm{~s}$ and then dissociated for $60 \mathrm{~s}$ at a rate of $30 \mu \mathrm{L} / \mathrm{min}$. The concentration (nM) of OVA was calculated by dividing the dry weight by $45000 \mathrm{Da}$. The injections were performed in the single-cycle kinetics mode. For regeneration, $4 \mathrm{M} \mathrm{MgCl}$ was injected for $30 \mathrm{~s}$. Kinetic constants were calculated from the sensorgrams using the $1: 1$ binding model of BIA evaluation software 4.1 (GE Healthcare). Measurements were made in triplicate.

EXiLE Assay RS-ATL8 cells $\left(5 \times 10^{4}\right.$ cells $/ 50 \mu \mathrm{L} /$ well $)$ were sensitized with $10 \mathrm{ng} / \mathrm{mL}$ anti-OVA $\mathrm{IgE}$ Abs and plated on a clear-bottom white 96-well plate (ViewPlate; PerkinElmer, Inc.). After overnight incubation, the cells were washed three times with PBS and then stimulated with OVA diluted in MEM containing $10 \%$ FCS $\left(50 \mu \mathrm{L} /\right.$ well) at $37^{\circ} \mathrm{C}$ under an atmosphere of $5.0 \% \mathrm{CO}_{2}$ for $3 \mathrm{~h}$. After stimulation, $50 \mu \mathrm{L}$ of luciferase substrate solution containing cell lysis reagent (ONE-Glo; Promega Corporation, Madison, WI, U.S.A.) was added to the cells, and chemiluminescence was measured using an EnVision multilabel plate reader (PerkinElmer, Inc.). Luciferase expression levels are represented as the fold increase of light units compared with the background expression (without stimulation) after subtraction of a blank control (without cells). The average value of measurements made in duplicate was used for analysis.

Western Blotting OVA was dissolved in $4 \times$ Laemmli sample buffer (Bio-Rad Laboratories, Hercules, CA, U.S.A.) with or without $5 \%$ 2-mercaptoethanol and boiled at $100^{\circ} \mathrm{C}$ for $3 \mathrm{~min}$. A total of $2.6 \mu \mathrm{g}$ per lane of OVA was resolved by $10 \%$ sodium dodecyl sulfate-polyacrylamide gel electrophoresis (SDS-PAGE) and transferred to a polyvinylidene fluoride (PVDF) membrane. After blocking with Blocking ONE reagent (Nacalai Tesque, Inc., Kyoto, Japan), the membrane was blotted with E-C1 and E-G5 Abs diluted 5000- and 500- fold overnight, respectively. Binding of $\operatorname{IgE}$ was detected with HRP-conjugated anti-mouse IgE (dilution, 1:15000; Bethyl Laboratories) and Clarity Western ECL substrate (Bio-Rad Laboratories).

Solid-Phase EXiLE Assay OVA diluted in $50 \mathrm{~mm}$ carbonate buffer ( $\mathrm{pH}$ 9.6) was plated on a high-binding 96-well plate (Corning Incorporated) for immobilization. After overnight incubation, OVA was washed with PBS and air-dried. Separately, RS-ATL8 cells were sensitized with $10 \mathrm{ng} / \mathrm{mL}$ antiOVA Abs overnight at $37^{\circ} \mathrm{C}$ under an atmosphere of $5 \% \mathrm{CO}_{2}$ and then the cells were collected by scraping, washed twice with sterile PBS, resuspended in culture medium, and then plated on the OVA-immobilized plate $\left(5.0 \times 10^{4}\right.$ cells $/ 50 \mu \mathrm{L} /$ well) and incubated for $3 \mathrm{~h}$ at $37^{\circ} \mathrm{C}$ under an atmosphere of $5 \% \mathrm{CO}_{2}$. After stimulation, the luciferase expression levels were measured as described before.

\section{RESULTS}

Binding Affinities Analyzed by ELISA E-C1 Ab, but not E-G5 Ab, has the potential to induce OVA-dependent degranulation of mast cells. ${ }^{11)}$ Before comparing the crosslinking abilities of these Abs, the binding capacity of E-C1 and E-G5 Abs to immobilized OVA protein was evaluated using ELISA. OVA was immobilized on a plastic 96-well plate, and the binding of anti-OVA IgE was detected with HRP-conjugated anti-IgE. As shown in Fig. 1, both E-C1 and E-G5 Abs bound


Fig. 1. ELISA Analysis of E-C1 and E-G5 Abs

OVA at the indicated concentration was immobilized on a microwell plate. The binding of E-C1 $\mathrm{Ab}$ (a) and E-G5 Ab (b) to immobilized OVA was detected with HRP-conjugated anti-mouse IgE. 

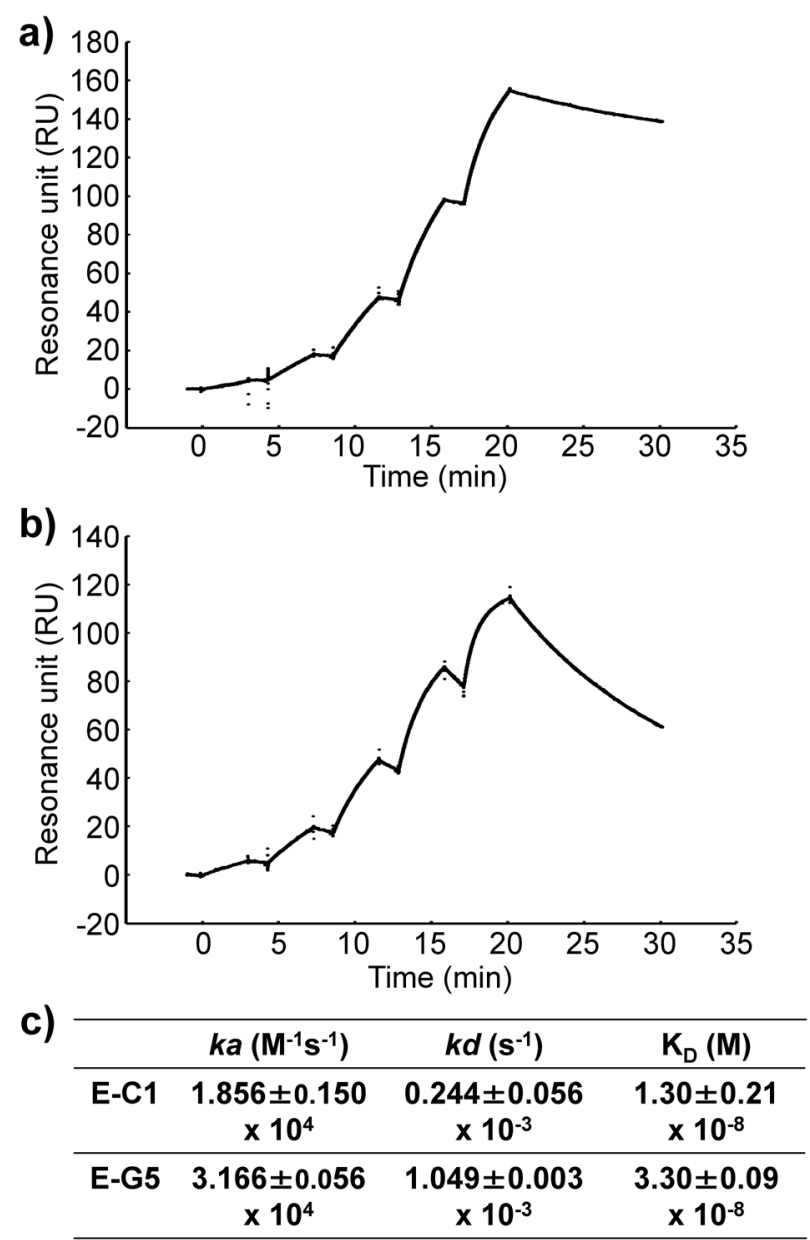

Fig. 2. SPR Analysis of E-C1 and E-G5 Abs

Representative sensorgrams of the binding between $10 \mu \mathrm{g} / \mathrm{mL}$ of $\mathrm{E}-\mathrm{C} 1 \mathrm{Ab}$ (a) or E-G5 Ab (b) and OVA $(0,8,24,72,216,648 \mathrm{~nm})$. (c) Kinetic constants \pm S.D. calculated from three independent experiments.

to OVA, although the binding curve of E-G5 Ab was slightly steeper than that of $\mathrm{E}-\mathrm{C} 1 \mathrm{Ab}$. These results suggest that these two monoclonal IgE Abs can directly bind at least to OVA immobilized in the solid phase, where the avidity of an antigen with an $\mathrm{Ab}$ is increased. ${ }^{13)}$ However, IgEs on the mast cells usually react to antigens dissolved in the liquid phase in vivo. Therefore, the lack of OVA-induced degranulating ability of E-G5 could be explained by different properties of the antigen in the liquid or solid phase, and thus, we analyzed the effects of the property differences in the following experiments.

SPR Analysis of Binding Affinities SPR analysis was performed to measure affinities between anti-OVA IgE Abs (E-C1 or E-G5) and OVA protein in the liquid phase. AntiOVA Abs were captured by pre-immobilized anti-mouse IgE on the sensor chip and then soluble OVA was injected as an analyte. As shown in Figs. 2a and b, OVA bound to and dissociated from E-C1 Ab more slowly than E-G5 Ab. As shown in Fig. 2c, the association and dissociation rate constants $\left(k_{\mathrm{a}}, k_{\mathrm{d}}\right.$, respectively) of the $\mathrm{E}-\mathrm{C} 1 \mathrm{Ab}$ were slightly lower than those of E-G5 Ab. Similarly, the equilibrium $k_{\mathrm{d}}$ of E-C1 was slightly lower than that of E-G5 $\left(1.30 \pm 0.21 \times 10^{-8} \mathrm{M} v\right.$. $3.30 \pm 0.09 \times 10^{-8} \mathrm{M}$, respectively), consistent with the results of ELISA analysis (Fig. 1). Hence, E-C1 and E-G5 Abs can bind to OVA both in the solid and liquid phases.

Crosslinking Abilities of E-C1 and E-G5 Abs Measured a)
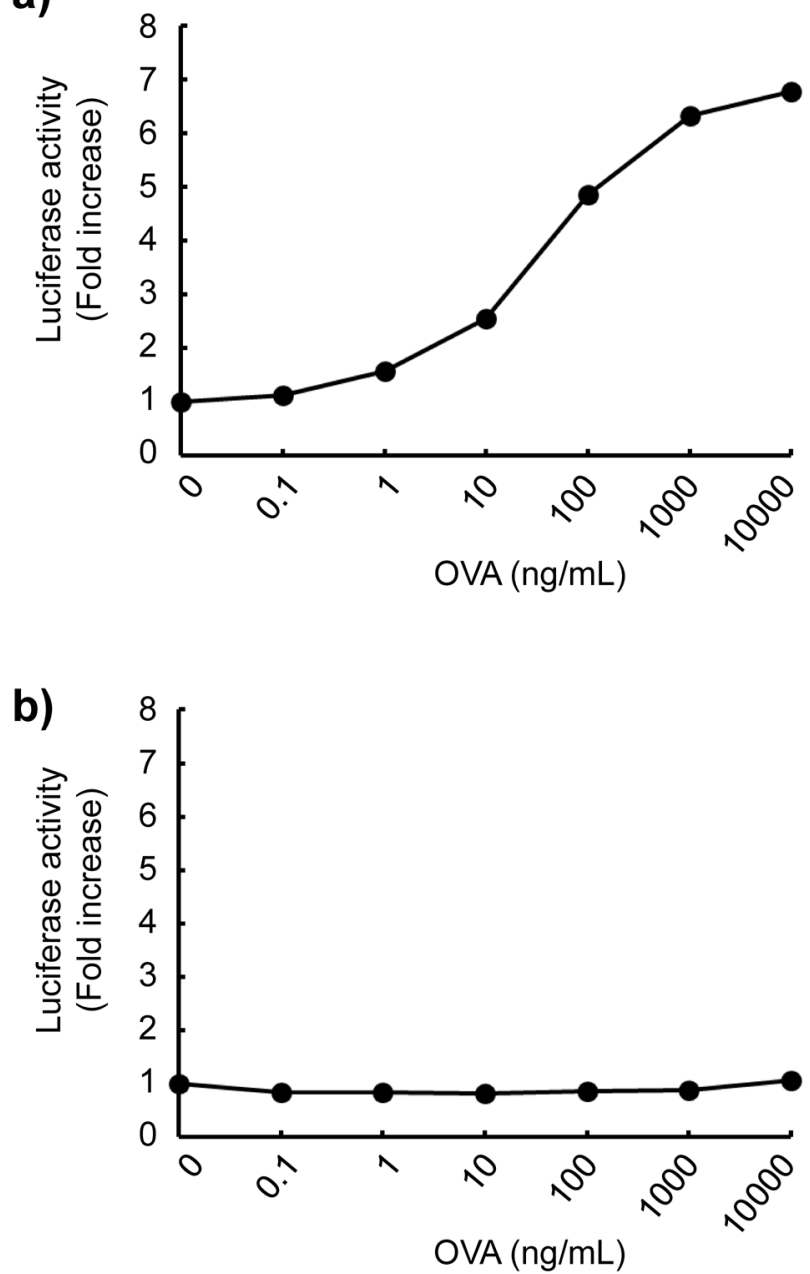

Fig. 3. EXiLE Analysis of E-C1 and E-G5 Abs

RS-ATL8 cells were sensitized with $10 \mathrm{ng} / \mathrm{mL}$ of E-C1 Ab (a) or E-G5 Ab (b) overnight and then stimulated with OVA at the indicated concentrations for $3 \mathrm{~h}$. Data are presented as fold increase, with the non-stimulated control designated as 1.0 .

by the EXiLE Test We next performed the EXiLE test, which is based on the crosslinking of FceRI, using the liquidphase antigen. After overnight sensitization of RS-ATL8 cells with $10 \mathrm{ng} / \mathrm{mL}$ of E-C1 or E-G5 Ab, OVA-induced luciferase expression was measured (Fig. 3). When sensitized with E-C1 $\mathrm{Ab}$, OVA markedly induced luciferase expression in a dosedependent manner (Fig. 3a). On the other hand, E-G5 Ab did not induce a luciferase response (Fig. 3b). These two Abs seemed to bind to the cells almost equally because stimulation of the cells with anti-mouse $\mathrm{IgE} \mathrm{Ab}$ led to similar levels of luciferase expression (10.8- and 9.2-fold for E-C1 and E-G5, respectively; not shown in Fig. 3). These results are consistent with previous data of another group and the manufacturer, which indicate that $\mathrm{E}-\mathrm{C} 1$ but not E-G5 Ab can induce mast cell degranulation. ${ }^{11,12)}$ However, it remains unclear why monoclonal E-C1 Ab can be crosslinked by OVA because there is no repeated sequences in the molecule. Recently, Ianeselli et al. showed that OVA forms dimers in solution using small-angle scattering. ${ }^{14)}$ If that is the case, monoclonal $\mathrm{IgE}$, such as E-C1, is able to be crosslinked by binding to an OVA dimer, which has two epitopes. Then, the question remains as to why E-G5 Ab, which can bind OVA in the liquid phase, cannot induce mast cell activation. 




Fig. 4. Western Blotting of OVA with E-C1 and E-G5 Abs

OVA was resolved on SDS-PAGE under non-reducing (a) or reducing (b) conditions and blotted with E-C1 and E-G5 Abs. M, molecular weight marker; CBB, Coomassie brilliant blue.

Western Blotting of OVA with Anti-OVA IgE Abs There are three possible explanations to why E-G5 Ab fails to induce mast cell activation. First, the binding affinity of E-G5 $\mathrm{Ab}$ may be too weak to form stable crosslinking, although it can bind to an immobilized antigen, as with an ELISA, which has a strong avidity. However, as shown in Fig. 2, the affinity of E-G5 Ab was comparable to that of E-C1 Ab; therefore, this possibility is very slim. Second, the epitope of E-G5 may be exposed only when OVA is immobilized on a hydrophobic plastic surface. However, this possibility is also unlikely because E-G5 Ab can directly bind to OVA in solution (Fig. 2). Finally, there is only one epitope on an OVA, even in its dimerized form. To determine the plausibility of this hypothesis, we performed Western blot analysis of OVA with the two IgE Abs. Briefly, OVA was resolved by SDS-PAGE under reducing or non-reducing conditions and was transferred to a PVDF membrane and blotted with IgE Abs. With regard to non-reduced proteins (Fig. 4a), E-C1 Ab bound to at least two bands at approximately 40 and $80 \mathrm{kDa}$, which corresponds to the monomer and dimer of OVA, respectively. ${ }^{15)}$ The smear band at $>80 \mathrm{kDa}$ seems to be an aggregation of OVA generated during the boiling procedure $\left(100^{\circ} \mathrm{C}, 3 \mathrm{~min}\right)$ before SDS-PAGE. Regarding reduced proteins, the OVA monomer appeared as more distinct bands at 40 and $42 \mathrm{kDa}$, which may correspond to free and phosphorylated/glycosylated OVA, respectively (Fig. 4b). These results strongly suggest that E-C1 $\mathrm{Ab}$ can bind to both the monomer and dimer of OVA, which is consistent with the results shown in Fig. 3. On the other hand, E-G5 Ab bound only to the component seen around $60-70 \mathrm{kDa}$ under non-reducing conditions (Fig. 4a). Although the molecular size is slightly smaller than that of the OVA dimer $(80 \mathrm{kDa})$, it is obviously different from that of the OVA monomer $(40 \mathrm{kDa})$. We found that E-G5 Ab did not show any immunoreactivity to the reduced OVA (Fig. 4b), suggesting that the $60-70 \mathrm{kDa}$ component under non-reduced conditions may correspond to the OVA dimer in a compact conformation with less abundance.

EXiLE Responses to Solid-Phase Antigens If E-G5 Ab binds to a single epitope that only exists on an OVA dimer,
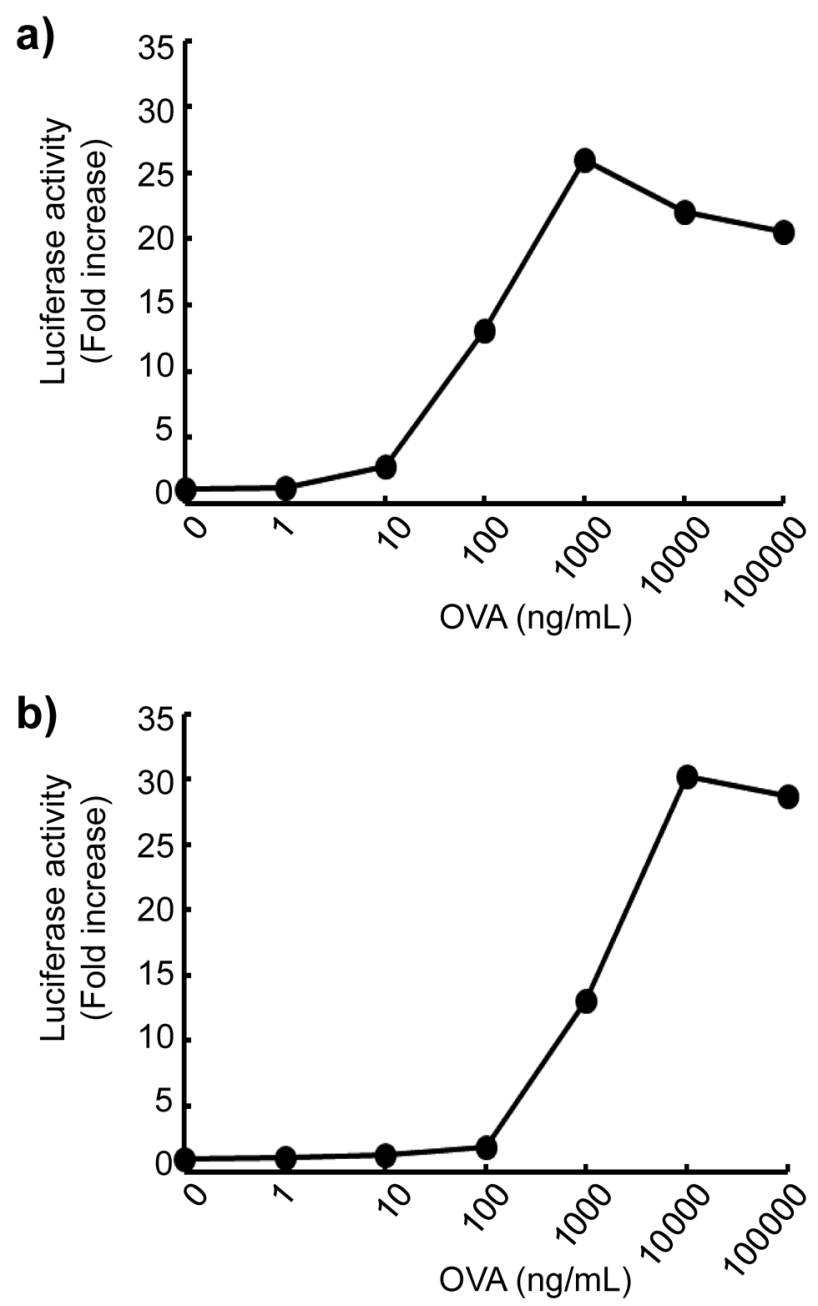

Fig. 5. Solid-Phase-EXiLE Analysis of E-C1 and E-G5 Abs

OVA at the indicated concentrations was immobilized on the plate. RS-ATL8 cells were sensitized with $10 \mathrm{ng} / \mathrm{mL}$ E-C1 Ab (a) or E-G5 Ab (b) overnight and stimulated by seeding on the OVA-immobilized plate. Data are presented as fold increase, with the non-stimulated control designated as 1.0.

immobilized OVA in the solid phase is expected to have adjacent multiple epitopes with a high avidity and be able to crosslink E-G5 Abs, unlike soluble OVA. To evaluate this possibility, OVA was immobilized on a plastic plate and reacted with anti-OVA Ab-sensitized RS-ATL8 cells (Fig. 5). As a result, not only E-C1 but also E-G5 Ab markedly induced the EXiLE response. The amplitude of the solid-phase EXiLE responses was much greater than that of the liquid phase (Fig. 3). This result clearly showed that immobilized OVA can crosslink E-G5 Ab (Fig. 5b), although it could not in the liquid phase (Fig. 3b), in accordance with our hypothesis that E-G5 Ab binds to an epitope on the OVA dimer.

\section{DISCUSSION}

The aim of this study was to evaluate several allergen detection methods based on immunochemical binding and receptor crosslinking using two OVA-specific mouse monoclonal IgEs. E-C1 Ab could not only bind to OVA but also induce receptor crosslinking, which leads to mast cell activation (Fig. 3a). On the other hand, although the binding of E-G5 Ab to OVA was detected by ELISA (Fig. 1b), this Ab did not induce mast cell activation (Fig. 3b), as expected from previous stud- 
ies. $^{11,12)}$ As mentioned earlier, OVA is thought to form dimers in solution, ${ }^{15)}$ which explains why $\mathrm{E}-\mathrm{Cl}$ monoclonal $\mathrm{Ab}$ induced crosslinking of FceRI, as shown in Fig. 3a. On the other hand, our Western blotting results strongly suggested that E-G5 Ab binds to epitopes that only exist on the OVA dimer (Fig. 4), suggesting that the E-G5 Ab cannot be crosslinked by the OVA dimer in the liquid phase, although the $\mathrm{Ab}$ can bind to the dimer in solution. The fact that the $k_{\mathrm{d}}$ of E-G5 Ab is greater than that of E-C1 (Fig. 2c) could be another reason for the lack of a stable crosslinking. However, OVA dimers immobilized in the solid phase acquired the crosslinking capacity of E-G5 Abs (Fig. 5b), probably through an increase in the avidity of the epitopes on the two OVA dimers.

As shown in Fig. 4a, the apparent molecular weight of the OVA dimer recognized by the E-G5 Ab under non-reduced conditions was slightly smaller than that of the E-C1 Ab-binding OVA dimer of $80 \mathrm{kDa}$. Considering that an OVA has four free cysteine residues and that non-reduced proteins do not necessarily migrate to an appropriate molecular size by SDSPAGE, the OVA dimer may have assumed a more compact conformation than the E-C1 Ab-binding OVA dimer. ${ }^{16)}$ The fact that the E-G5-binding structure was sensitive to reduction (Fig. 4b) also supports this hypothesis.

From a clinical point of view, allergen-specific IgE with specificity similar to the E-G5 Ab would result in a false positive on immunochemical allergy tests, such as the CAP test. The findings of this study re-emphasized the difference in the physicochemical nature of allergens in the liquid and solid phases. Considering that most of the allergens are soluble proteins, it seems important to evaluate the interaction of $\operatorname{IgE}$ and allergen in the liquid phase, as far as possible. In this context, similar caution should be exercised when applying the results of new techniques, such as multi-dimensional $\operatorname{IgE}$ blotting for allergenomics, and new allergen discovery, ${ }^{17)}$ such as allergen microarrays for high-throughput clinical diagnosis using basophils. ${ }^{18)}$ After immunochemical measurements like these, it seems that it is still necessary to confirm the ability of FceRI crosslinking. These results suggest that the EXiLE test is a promising method to confirm the clinical relevance of $\operatorname{IgE}$ binding to soluble allergens.

Acknowledgments This study was supported by JSPS KAKENHI Grant Numbers 25460082, 15K19168 and the Japan Agency for Medical Research and Development (AMED).

Conflict of Interest The authors declare no conflict of interest.

\section{REFERENCES}

1) van Ree R, Aalberse RC. Specific IgE without clinical allergy. $J$. Allergy Clin. Immunol., 103, 1000-1001 (1999).

2) Mari A, Iacovacci P, Afferni C, Barletta B, Tinghino R, Di Felice G, Pini C. Specific IgE to cross-reactive carbohydrate determinants strongly affect the in vitro diagnosis of allergic diseases. J. Allergy Clin. Immunol., 103, 1005-1011 (1999).

3) Blank U, Ra C, Miller L, White K, Metzger H, Kinet JP. Complete structure and expression in transfected cells of high affinity $\operatorname{IgE}$ receptor. Nature, 337, 187-189 (1989).

4) Gleich GJ, Yunginger JW. The radioallergosorbent test: a method to measure IgE antibodies, IgG blocking antibodies, and the potency of allergy extracts. Bull. N. Y. Acad. Med., 57, 559-567 (1981).

5) Axen RDH, Kober A, Yman L. Proceedings of a clinical workshop: $\mathrm{IgE}$ antibodies and the pharmacia CAP system in allergy diagnosis. A new laboratory diagnostic system applied to allergy testing. (Johansson SGO ed.) Pharmacia Publication Sweden, pp. 3-5 (1988).

6) Nakamura R, Uchida Y, Higuchi M, Nakamura R, Tsuge I, Urisu A, Teshima R. A convenient and sensitive allergy test: IgE crosslinking-induced luciferase expression in cultured mast cells. Allergy, 65, 1266-1273 (2010).

7) Nakamura R, Ishiwatari A, Higuchi M, Uchida $Y$, Nakamura R, Kawakami H, Urisu A, Teshima R. Evaluation of the luciferase assay-based in vitro elicitation test for serum IgE. Allergol. Int., 61, 431-437 (2012).

8) Nakamura R, Nakamura R, Adachi R, Itagaki Y, Fukutomi Y, Teshima R. Evaluation of allergenicity of acid-hydrolyzed wheat protein using an in vitro elicitation test. Int. Arch. Allergy Immunol., 160, 259-264 (2013).

9) Nakamura R, Nakamura R, Sakai S, Adachi R, Hachisuka A, Urisu A, Fukutomi Y, Teshima R. Tissue transglutaminase generates deamidated epitopes on gluten, increasing reactivity with hydrolyzed wheat protein-sensitized IgE. J. Allergy Clin. Immunol., 132, 1436-1438 (2013).

10) Falcone FH, Alcocer MJ, Okamoto-Uchida Y, Nakamura R. Use of humanized rat basophilic leukemia reporter cell lines as a diagnostic tool for detection of allergen-specific IgE in allergic patients: time for a reappraisal? Curr. Allergy Asthma Rep., 15, 67 (2015).

11) "Allergy-Allergenic IgE Monoclonal Antibodies/OVA.": 〈https://www. chondrex.com/animal-models/allergenic-Ige-monoclonal-antibodies.), cited 18 April, 2016.

12) Mizutani N, Goshima H, Nabe T, Yoshino S. Establishment and characterization of a murine model for allergic asthma using allergen-specific IgE monoclonal antibody to study pathological roles of IgE. Immunol. Lett., 141, 235-245 (2012).

13) Vorup-Jensen T. On the roles of polyvalent binding in immune recognition: perspectives in the nanoscience of immunology and the immune response to nanomedicines. Adv. Drug Deliv. Rev., 64, 1759-1781 (2012).

14) Ianeselli L, Zhang F, Skoda MW, Jacobs RM, Martin RA, Callow S, Prevost S, Schreiber F. Protein-protein interactions in ovalbumin solutions studied by small-angle scattering: effect of ionic strength and the chemical nature of cations. J. Phys. Chem. B, 114, 37763783 (2010)

15) Ishimaru $T$, Ito $K$, Tanaka M, Matsudomi N. Thermostabilization of ovalbumin by alkaline treatment: Examination of the possible roles of D-serine residues. Protein Sci., 19, 1205-1212 (2010).

16) Nakamura R, Ishimaru M. Changes in the shape and surface hydrophobicity of ovalbumin during its transformation to $s$-ovalbumin. Agric. Biol. Chem., 45, 2775-2780 (1981).

17) Nakamura $R$, Satoh $R$, Nakamura $R$, Shimazaki $T$, Kasuga M, Yamaguchi-Shinozaki K, Kikuchi A, Watanabe KN, Teshima R. Immunoproteomic and two-dimensional difference gel electrophoresis analysis of Arabidopsis dehydration response element-binding protein 1A (DREB1A)-transgenic potato. Biol. Pharm. Bull., 33, 1418-1425 (2010).

18) Lin J, Renault N, Haas H, Schramm G, Vieths S, Vogel L, Falcone FH, Alcocer MJ. A novel tool for the detection of allergic sensitization combining protein microarrays with human basophils. Clin. Exp. Allergy, 37, 1854-1862 (2007). 